\title{
PILOT STUDY OF THE RELATIONSHIP BETWEEN VOCABULARY ABILITY AND LEARNING PERFORMANCE FOR UNFAMILIAR KNOWLEDGE IN ONLINE VIDEO CLASSES
}

\author{
Kaori Tamura \\ Department of Information and Systems Engineering, Faculty of Information Engineering, \\ Fukuoka Institute of Technology \\ 3-30-1 Wajiro-higashi, Higashi-ku, Fukuoka 811-0295, Japan
}

\begin{abstract}
The COVID-19 pandemic has led to the introduction of online courses in higher education worldwide, including online video courses. In a video course, it is difficult to predict which students cannot follow a course that provides unfamiliar knowledge. Therefore, this pilot study investigated the relationship between learning performance of unfamiliar knowledge and Japanese vocabulary ability. A total of 59 college students participated in this pilot study. After their Japanese proficiency levels were measured by their grammatical and vocabulary abilities, they took a video course about the "Structure of Brain" (as an unfamiliar topic). The students took a short pre- and post-lecture knowledge test. The results of this study revealed that students with a high level of Japanese vocabulary ability showed higher learning performance for unfamiliar knowledge delivered via the video lecture. This pilot study contributes to predicting students' online learning performance based on student properties.
\end{abstract}

\section{KEYWORDS}

e-Learning, Video Course, Vocabulary, Prediction of Learning Performance

\section{INTRODUCTION}

In 2020, many universities were forced to provide online classes instead of conventional face-to-face classes due to the COVID-19 pandemic. Although the importance and effectiveness of remote education have been discussed in previous studies, most educational institutes provided conventional face-to-face classes before the pandemic. The pandemic forced instructors to make a sudden shift to online learning platforms. The use of a combination of online and face-to-face learning styles will be increasingly used even after the COVID-19 pandemic has subsided.

Lectures can be delivered remotely in several ways. Recorded video lectures enable students to learn flexibly, with no restrictions regarding time and place; however, they also have some drawbacks. For instructors, it is difficult to judge whether learners understood the content of the recorded video lectures because of a lack of simultaneous feedback and immediate interaction in the medium. When teaching new knowledge belonging to an unfamiliar field to learners, an instructor will fail to predict learners' understanding in recorded video lectures.

In this study, we aimed to explore the parameters of learners that helped predict their learning performance after watching a video lecture that provided unfamiliar knowledge. These parameters will enable the appropriate pacing of video course lectures despite the lack of simultaneous feedback from learners. In this study, we focused on learners' grammatical and vocabulary abilities of the language used for instruction, that is, Japanese. Existing studies have reported that learners' vocabulary is related to reading comprehension (Dickinson and McCabe, 2001; Ouellette, 2006), that can affect their knowledge acquisition. We hypothesized that grammatical and vocabulary abilities could be candidate parameters to predict learners' learning performance in video courses. 


\section{METHOD}

All procedures of this study were performed in accordance with the guidelines of the ethics committee of Fukuoka Institute of Technology. A total of 59 undergraduate students of Fukuoka Institute of Technology participated in the study. They were students of the faculty of Information Engineering and took a class on "human biological system." All the participants provided informed consent in accordance with the Declaration of Helsinki. All the participants, excepting one student, were native Japanese speakers. Two participants declined to participate in the experiments after their written consent statements were obtained. One participant's responses were not considered in the final analysis because he was unable to complete his test responses within the stipulated time (over mean response time $+2 \mathrm{SD}$ ). Finally, the data from 56 participants were analyzed.

The participants were asked to participate in two online experiments. Experiment 1 measured their Japanese language skills, and Experiment 2 measured their understanding and acquisition of unfamiliar knowledge delivered using an online video lecture. Their responses were collected using Microsoft Forms (Microsoft Corporation, Redmond, WA, USA).

\subsection{Experiment 1: Japanese Language Skills}

To measure the participants' Japanese language skills, we used the Grade I of the Japanese Language Examination (conducted by the Japanese Language Examination Committee), that is designed for all Japanese speakers, including native speakers. The Grade I of the examination is equivalent to the advanced level of business Japanese, with a pass rate of $22.3 \%$ in 2020. In this experiment, we used 10 quizzes for measuring participants' vocabulary ability and 10 quizzes for measuring their grammatical ability; these quizzes were derived from the abovementioned examination. Each quiz contained multiple-choice questions, with a correct response to be chosen from four options.

\subsection{Experiment 2: Video Lecture}

The participants watched a lecture movie about the human brain anatomy, a part of the class on human biological system, and took a short pre- and post-lecture knowledge test. The questions in the tests were related to the content of the lecture movie. The length of the movie was approximately 15 min, and it consisted of slides about the learning content with the instructor's voiceover. The instructor did not reveal her face.

After watching the movie, the participants were also asked about their perceptions of cognitive load and situational interest (Wang, Antonenko and Dawson, 2020) (ranked from 1 to 7). Cognitive load is classified into overall load and three subtypes: intrinsic load, extraneous load, and germane load (Wang, Antonenko and Dawson, 2020). The overall load was assessed by the participants' self-report of the amount of mental effort invested in watching the video (Paas, 1992). The intrinsic load was measured by the students' ratings of content difficulty, and it has been assumed in this study that the intrinsic load was affected by the content and not by the instructional design (Cierniak, Scheiter and Gerjets, 2009). In this experiment, the intrinsic load was measured by the students' ratings for several difficult terminologies in the movie, texts in slides, auditory inputs (information from the narration). The extraneous load was measured by the students' ratings of how easy it was to learn from the online movie. The germane load was measured by the participants' self-reported level of concentration while watching the movie. Situational interest was measured by students' ratings for the statements "I am interested in the content" and "I am willing to learn new content related to the movie content".

\section{RESULTS}

\subsection{Learning Performance after the Video Lecture}

We confirmed the students' prior knowledge related to learning content and learning performance from the lecture movie. The rate of correct responses between the pre- and post-lecture knowledge test was compared. 
The mean \pm standard error $(\mathrm{SEM})$ of the rate of correct responses pre-lecture was $0.24 \pm 0.025$, and that of post-lecture was $0.47 \pm 0.033$. A paired t-test showed a significant difference between pre- and post-lecture $(\mathrm{t}(55)=-6.9, \mathrm{p}<0.001)$ rates. The statistical results indicate that the rate of correct responses significantly increased after the students watched the movie lecture.

\subsection{Japanese Language Ability and Learning Performance}
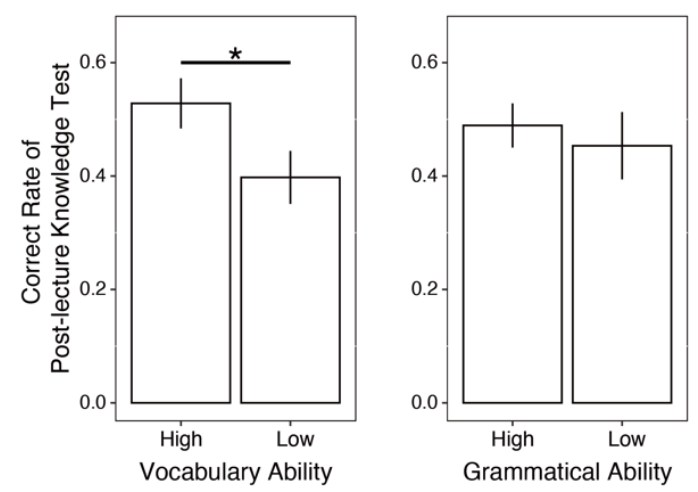

Figure 1. Mean and SEM of the correct rate of post-lecture knowledge test. The participants were divided by vocabulary ability scores (left) or grammatical ability scores (right) measured in Experiment $1(*$ : $<<0.05)$

We analyzed the relationship between the participants' Japanese language ability and post-lecture knowledge test scores (Fig. 1). Based on a balanced median split on the vocabulary ability scores as measured in Experiment 1, a high-vocabulary and a low-vocabulary ability group were formatted. The high-vocabulary ability group showed significantly higher scores on the post-lecture knowledge test than the low vocabulary ability group $(\mathrm{t}(51)=2.0, \mathrm{p}=0.047)$.

Based on a balanced median split on the grammatical ability scores as measured in Experiment 1, a high-grammatical and low-grammatical ability group were formatted. For grammatical ability, there were no significant differences in the post-lecture knowledge test scores between the high and low grammatical ability groups $(\mathrm{t}(40)=0.50, \mathrm{p}=0.62)$.

\subsection{Learner Perceptions and Learning Performance}

We analyzed the relationships between the participants' learning performance and perceptions. There was no significant correlation between the rate of correct responses post-lecture and the four types of cognitive load: overall load, intrinsic load, extraneous load, and germane load (Table 1). Regarding the situational interest, there was a significant but very weak correlation between the post-lecture learning performance and the ratings regarding the following statement: "I am willing to learn new content related to the movie content."

Table 1. Correlation coefficients between perception ratings and the rate of correct responses in the post-lecture test.

All the correlation coefficients were calculated from several ratings and the rate of correct responses post-lecture.

P-values indicated the significances of the correlation coefficients. Asterisk indicates significance level $(*: \mathrm{p}<0.05)$

\begin{tabular}{llll}
\hline Perception rating & Subtypes & Correlation coefficient & p-value \\
\hline Overall load & & 0.13 & 0.35 \\
Intrinsic loads & Difficulty in terminology & 0.094 & 0.50 \\
& The difficulty of texts in slides & 0.13 & 0.36 \\
& The difficulty of auditory inputs & 0.012 & 0.93 \\
Extraneous load & & 0.086 & 0.53 \\
Germane load & & 0.23 & 0.083 \\
Situational interest & Interested in the lecture & 0.19 & 0.18 \\
& Willing to learn more & 0.28 & $0.041^{*}$ \\
\hline
\end{tabular}




\section{DISCUSSION}

In this study, we aimed to investigate the relationship between undergraduate students' language abilities and learning performance of unfamiliar knowledge. From our results, we can suggest that vocabulary ability can affect students' learning performance of unfamiliar knowledge; however, we could not find any significant effect of grammatical ability on students' learning performance of unfamiliar knowledge.

The participants with higher scores in the Japanese vocabulary ability test showed a higher level of knowledge acquisition from the video lecture. Thus, vocabulary ability seems to relate to learning unfamiliar knowledge, although the vocabulary items asked in the Japanese language examination did not include terms related to the structure of the brain. We can assume that high vocabulary ability can support the acquisition of unfamiliar knowledge. The relationship between vocabulary skills and learning performance (Cain, Oakhill, \& Lemmon, 2004) has been discussed previously; however, these studies investigated elementary and junior high school students. We suggest the possibility that vocabulary skills could affect undergraduate students' acquisition of knowledge from video courses.

The limitations of this study need to be discussed. In this study, we investigated only one topic, the "Structure of Brain," as it belonged to a knowledge field unfamiliar to the participants. To generalize the findings of this study, more learning contents should be assessed. We could not determine whether the scores reflected the test difficulty or the participants' low motivation for the experiment. For further investigation, it is necessary to assess participants' language proficiency levels with a lower grade of Japanese Language Examination. This study focused on learning with online video lectures, but there is a need to investigate the results of this study in other environments, including face-to-face lessons.

This pilot study showed a high probability that students with high vocabulary ability can learn new knowledge from online video courses efficiently. The results of this study will help instructors predict learners' performance in online education.

\section{ACKNOWLEDGEMENT}

This work was supported by a JSPS Grant-in-Aid for Early-Career Scientists (Grant Number 19K14319).

\section{REFERENCES}

Cain, K., Lemmon, K. and Oakhill, J. (2004) 'Individual differences in the inference of word meanings from context: The influence of reading comprehension, vocabulary knowledge, and memory capacity', Journal of Educational Psychology, 96(4), pp. 671-681. doi: 10.1037/0022-0663.96.4.671.

Cierniak, G., Scheiter, K. and Gerjets, P. (2009) 'Explaining the split-attention effect: Is the reduction of extraneous cognitive load accompanied by an increase in germane cognitive load?', Computers in Human Behavior. Pergamon, 25(2), pp. 315-324. doi: 10.1016/j.chb.2008.12.020.

Dickinson, D. K. and McCabe, A. (2001) 'Bringing it all together: The multiple origins, skills, and environmental supports of early literacy', Learning Disabilities Research and Practice, 16(4), pp. 186-202. doi: 10.1111/0938-8982.00019.

Ouellette, G. P. (2006) 'What's meaning got to do with it: The role of vocabulary in word reading and reading comprehension', Journal of Educational Psychology, 98(3), pp. 554-566. doi: 10.1037/0022-0663.98.3.554.

Paas, F. G. W. C. (1992) 'Training strategies for attaining transfer of problem-solving skill in statistics: A cognitive-load approach', Journal of Educational Psychology, 84(4), pp. 429-434. doi: 10.1037/0022-0663.84.4.429.

Wang, J., Antonenko, P. and Dawson, K. (2020) 'Does visual attention to the instructor in online video affect learning and learner perceptions? An eye-tracking analysis', Computers and Education. Elsevier Ltd, 146, p. 103779. doi: 10.1016/j.compedu.2019.103779 